\title{
Baixa autoestima situacional em gestantes: uma análise de acurácia
}

\author{
Situational low self-esteem in pregnant women: an analysis of accuracy \\ Baja autoestima situacional en gestantes: un análisis de acurácia
}

\section{Joyce Carolle Bezerra Cavalcante', Vanessa Emille Carvalho de Sousa", Marcos Venícios de Oliveira Lopes'II \\ ' Universidade Federal do Ceará, Faculdade de Farmácia Odontologia e Enfermagem, Curso de Graduação em Enfermagem (Graduada). Fortaleza-CE, Brasil. \\ "Universidade Federal do Ceará, Faculdade de Farmácia Odontologia e Enfermagem, Departamento de Enfermagem, Programa de Pós-Graduação em Enfermagem (Doutoranda). Fortaleza-CE, Brasil. \\ I' Universidade Federal do Ceará, Faculdade de Farmácia Odontologia e Enfermagem, Departamento de Enfermagem, Programa de Pós-Graduação em Enfermagem. Fortaleza-CE, Brasil.}

Submissão: 12-04-2011 Aprovação: 01-02-2013

\section{RESUMO}

Para investigar a acurácia de características definidoras do diagnóstico Baixa autoestima situacional, realizou-se um estudo transversal, com 52 gestantes atendidas num centro de atendimento familiar. Utilizou-se a taxonomia da NANDA-I e a escala de Rosenberg. O diagnóstico esteve presente em $32,7 \%$ das gestantes e todas as características apresentaram associação estatística com o diagnóstico, exceto "Relata verbalmente desafio situacional ao seu próprio valor". As características com maior sensibilidade foram "Comportamento indeciso" e "Expressões de desamparo" (82,35\%). Já as características "Expressões de sentimento de inutilidade" e "Relata verbalmente desafio situacional atual ao seu próprio valor" apresentaram maior especificidade $(94,29 \%)$. Tais resultados podem contribuir com a prática de enfermagem, pois a identificação de características acuradas é fundamental para uma inferência segura.

Descritores: Diagnóstico de Enfermagem; Autoimagem; Gravidez.

\begin{abstract}
To investigate the accuracy of defining characteristics of Situational low self-esteem we developed a cross-sectional study, with 52 pregnant women assisted in a family centre. The NANDA-I taxonomy was used as well as the Rosenberg's scale. The diagnosis was present in $32.7 \%$ of the sample and all characteristics presented statistical significance, except "Reports verbally situational challenge to its own value". The characteristics "Indecisive behavior" and "Helplessness expressions" had 82.35\% of sensitivity. On the other hand, the characteristics "Expression of feelings of worthlessness" and "Reports verbally situational challenge to its own value" were the more specific, with $94.29 \%$ of specificity. These results can contribute with the nursing practice because the identification of accurate characteristics is essential to a secure inference.
\end{abstract}

Key words: Nursing Diagnosis; Self Concept; Pregnancy.

\section{RESUMEN}

Para investigar la acurácia de características definidoras del diagnostico Baja autoestima situacional, desarrollamos un estudio transversal, con 52 gestantes atendidas en un centro familiar. La taxonomía NANDA-I y la escala de Rosenberg fueron utilizadas. El diagnostico estaba presente en $32.7 \%$ de las mujeres y todas las características fueron estadísticamente significativas, a menos que "Informa verbalmente desafío situacional a su valor propio". Las características "Comportamiento incierto" y "Expresiones de abandono" tuvieron $82.35 \%$ de sensibilidad. Las características "Expresiones de sentimientos de inutilidad" y "Informa verbalmente desafío situacional a su valor propio" fueron las más específicas, con 94.29\% de especificidad. Estos resultados pueden contribuir con la práctica de enfermería porque la identificación de características acuradas es esencial para una inferencia diagnostica segura.

Palabras clave: Diagnóstico de Enfermería; Autoimagen; Embarazo. 


\section{INTRODUÇÃO}

A gestação é um período do ciclo vital que repercute significativamente sobre a autopercepção feminina e sobre sua relação com o mundo. As diversas modificações físicas e funcionais decorrentes da gravidez, sobretudo a partir do segundo trimestre, representam motivos de satisfação ou de insatisfação, de acordo com a concepção de cada gestante ${ }^{(1)}$.

De uma maneira geral, os sentimentos negativos costumam aumentar com o avanço da gestação. Além disso, a instabilidade emocional resultante das mudanças hormonais pode repercutir em uma situação geradora de estresse. Para uma melhor adaptação da mulher a essas transformações e ao novo papel de mãe é necessária a aceitação do status de gestante, um fenômeno presente entre mulheres com autoestima elevada ${ }^{(2)}$. Por outro lado, alterações psicopatológicas podem desencadear alterações fisiológicas ${ }^{(3)}$.

A consulta de pré-natal é um momento oportuno para a avaliação da autoestima e do autoconceito da gestante, pois nela podem ser abordados problemas reais e potenciais associados ao período gestacional. Entretanto, a investigação destes aspectos junto a gestantes tem sido deficiente, e um dos motivos talvez seja a subjetividade das respostas psicológicas que dificulta o desenvolvimento de planos de cuidados específicos.

Uma abordagem específica da Enfermagem relacionada à autoestima é a avaliação da presença do diagnóstico de enfermagem Baixa autoestima situacional, contemplado pela Taxonomia II da NANDA Internacional (NANDA-I). A Baixa autoestima situacional e o Risco de baixa autoestima situacional reportam-se a sentimentos negativos sobre si e suas próprias capacidades em resposta a uma situação atual ${ }^{(4)}$.

Alguns estudos com foco em diagnósticos de enfermagem em gestantes têm sido desenvolvidos ${ }^{(5-6)}$. Contudo, ainda há uma carência de estudos que retratem as respostas psicológicas da mulher frente às alterações decorrentes da gestação. Estudos voltados para a acurácia de características definidoras contribuem para o desenvolvimento de uma melhor inferência diagnóstica podendo repercutir diretamente na qualidade da assistência.

Não foi encontrada na literatura uma tecnologia para avaliar as características definidoras do diagnóstico citado, demonstrando a escassez de pesquisas na enfermagem relacionadas à autoestima e aos seus respectivos diagnósticos. No entanto, a Rosenberg Self-Esteem Scale (RSES) foi considerada pertinente para este propósito, pois se trata de um dos instrumentos mais utilizados para a avaliação da autoestima global ${ }^{(7)}$.

A RSES é uma escala do tipo Likert, composta por dez afirmativas com quatro opções de resposta: concordo totalmente, concordo, discordo e discordo totalmente. Sua finalidade é classificar o nível e autoestima a partir de uma autoavaliação do indivíduo acerca dos sentimentos negativos e positivos a respeito de si mesmo, ressaltando questões de satisfação pessoal, autodepreciação, percepção de qualidades, competência, orgulho por si, autovalorização, respeito e sentimento de fracasso $^{(8)}$. A pontuação total da escala pode oscilar entre 10 e 40, de maneira que quanto maior a pontuação, maior a autoestima.

Desta forma, este estudo teve como objetivo identificar a acurácia das características definidoras do diagnóstico de enfermagem Baixa autoestima situacional e verificar a sensibilidade e especificidade da RSES para a identificação do mesmo em gestantes.

\section{MÉTODOS}

Estudo transversal desenvolvido junto a gestantes acompanhadas em um centro de atendimento familiar, pertencente a uma universidade federal. Este serviço oferece acompanhamento pré-natal e prevenção ginecológica às mulheres da área adstrita realizados por enfermeiros.

Para o cálculo do tamanho amostral utilizou-se a fórmula para populações finitas, partindo de uma população de 208 gestantes cadastradas na unidade de saúde (número levantado mediante consulta aos registros). Atribuíram-se coeficiente de confiança de $90 \%$, erro amostral de $10 \%$ e prevalência do evento de 50\% devido à impossibilidade de se definir a quantidade de pessoas com o diagnóstico em estudo. Obteve-se um número de 52 gestantes na amostra, selecionadas de forma consecutiva à medida que buscavam atendimento no centro onde o estudo foi desenvolvido e atendiam aos critérios de seleção.

O estudo utilizou como critérios de inclusão o cadastro da gestante no centro e período gestacional a partir do segundo trimestre. Os critérios de exclusão foram: desorientação ou sequelas neurológicas e a história de depressão autorrelatada. A anuência ao estudo foi concedida, após o esclarecimento dos objetivos da pesquisa, mediante a assinatura do Termo de Consentimento Livre e Esclarecido. O estudo foi aprovado pelo Comitê de Ética da instituição responsável pela pesquisa, sob o protocolo 168/09.

A coleta de dados foi realizada por meio de entrevista em sala privativa. Utilizou-se um instrumento desenvolvido com base na NANDA-I ${ }^{(4)}$ e na $\operatorname{RSES}^{(9)}$. Vale ressaltar que esta escala já foi submetida à adaptação transcultural ${ }^{(8)}$ e possui confiabilidade comprovada em estudos para a avaliação da autoestima de gestantes ${ }^{(10-11)}$.

Utilizou-se a RSES para a identificação das características definidoras propostas pela NANDA-I, correlacionando-se cada característica a uma afirmativa da escala, com exceção de "Comportamento indeciso" e "Expressões de desamparo", que foram analisadas isoladamente. Das dez afirmativas presentes na RSES, foram selecionadas cinco, que tinham maior correlação com as características definidas pela NANDA-I para o diagnóstico de interesse (Tabela 1).

Considerou-se que as características definidoras estavam presentes quando a entrevistada concordava ou concordava totalmente com a respectiva afirmativa na escala de Rosenberg, com exceção de "Relata verbalmente desafio situacional atual ao seu próprio valor", que foi categorizada como presente nos casos de discordância ou discordância total em relação à afirmativa correspondente na escala.

A avaliação da característica "Comportamento indeciso" foi executada por meio da pergunta: "Você tem dificuldade para tomar decisões ultimamente?", enquanto "Expressões de desamparo" foi avaliada perguntando-se: "Você se sente desamparada ou sem alguém para te apoiar nas dificuldades?". 
Tabela 1 - Correspondência entre itens da escala de Rosenberg e características definidoras do diagnóstico Baixa autoestima situacional.

\begin{tabular}{ll}
\hline Características definidoras & Afirmativas da escala de Rosenberg \\
\hline 1. Avaliação de si mesmo como incapaz de lidar com situações ou eventos & "Ás vezes eu acho que não presto para nada" \\
2. Comportamento indeciso & - \\
3. Comportamento não assertivo & \\
4. Expressões de desamparo & "Às vezes eu me sinto inútil" \\
5. Expressões de sentimento de inutilidade & "Eu sinto que sou uma pessoa de valor, pelo menos no \\
6. Relata verbalmente desafio situacional atual ao seu próprio valor & mesmo nível do que as outras" \\
7. Verbalizações autonegativas & "Eu sinto que não tenho muito do que me orgulhar" \\
\hline
\end{tabular}

Ambas as características foram consideradas presentes quando a resposta era "sim" ou "às vezes".

Além da aplicação da RSES, o instrumento contemplou dados de identificação e dados obstétricos. Com base nas recomendações para análise da acurácia diagnóstica ${ }^{(12-13)}$, os resultados da coleta foram analisados pelos autores do estudo, cumprindo as etapas de raciocínio diagnóstico para a definição da presença do diagnóstico de interesse bem como de suas características definidoras.

Os dados foram analisados com apoio do software R versão 2.12. Dados sócio-demográficos, obstétricos e as respostas referentes às características definidoras de interesse foram considerados variáveis independentes, enquanto o diagnóstico de enfermagem em estudo foi considerado variável desfecho, a partir dos dados relacionados à pontuação final da escala e pela avaliação do especialista.

Procedeu-se à apresentação dos resultados em tabelas, sendo utilizado o teste de Kolmogorov-Smirnov para verificação da normalidade. Realizou-se análise descritiva com a apresentação de percentuais, medidas de tendência central e de dispersão. $\mathrm{Na}$ análise inferencial foram aplicados os testes de Qui-quadrado, para verificar a associação entre as variáveis, ou o teste de Fisher, quando as frequências esperadas eram menores que cinco. Para estimativa da magnitude do efeito foram calculadas as razões de prevalência e seus intervalos de confiança de $95 \%$. As análises de diferença de média e mediana foram realizadas utilizando-se o teste t e o teste de
Mann-Whitney, respectivamente. Medidas de sensibilidade, especificidade, valor preditivo, razão de verossimilhança, odds ratio diagnóstica, acurácia e área sob a curva ROC das características definidoras do diagnóstico de interesse foram analisados para a investigação da acurácia, sendo definido o ponto de corte de $80 \%$. O nível de significância para as inferências estatísticas foi estabelecido em 0,05.

\section{RESULTADOS}

A média de idade das mulheres avaliadas foi de 22,21 anos (DP $=4,88)$, sendo que $28,8 \%$ delas eram adolescentes. Em relação ao estado civil, $88,5 \%$ eram casadas ou moravam com o companheiro. Quanto à escolaridade, foi obtida uma mediana de 10 anos de estudo, contados a partir do primeiro ano do ensino fundamental, correspondente a ensino médio incompleto. A renda per capita média foi de $\mathrm{R} \$ 186,02$ (DP $=$ 119,29 ) e $82,7 \%$ das gestantes estavam desempregadas.

Em relação aos dados obstétricos, a maioria das mulheres $(53,8 \%)$ tinha menos de 26 semanas de gestação, sendo a média de 25,9 semanas. A altura uterina média foi de 25,7 $\mathrm{cm}$. A gravidez foi referida como não planejada em $63,5 \%$ dos casos. Do total, $46,1 \%$ eram primigestas e $63,4 \%$ fizeram pelo menos duas consultas de pré-natal (mediana de duas consultas). O número de multigestas correspondeu a $53,85 \%$ das mulheres e o de nulíparas a $51,9 \%$. Sete gestantes $(13,4 \%)$ referiram aborto anterior sendo, um destes, induzido $(14,2 \%)$.

Tabela 2 - Prevalência das características definidoras e do diagnóstico Baixa autoestima situacional em gestantes.

\begin{tabular}{|c|c|c|}
\hline Características definidoras & $N^{\circ}$ & $\%$ \\
\hline 1. Comportamento indeciso & 29 & 55,8 \\
\hline 2. Comportamento não assertivo & 12 & 23,1 \\
\hline 3. Expressões de desamparo & 21 & 40,4 \\
\hline 4. Expressões de sentimento de inutilidade & 15 & 28,8 \\
\hline 5. Relata verbalmente desafio situacional atual ao seu próprio valor & 03 & 5,8 \\
\hline 6. Verbalizações autonegativas & 17 & 32,7 \\
\hline Baixa autoestima situacional & $\mathbf{N}^{\circ}$ & $\%$ \\
\hline Presente & 17 & 32,7 \\
\hline Ausente & 35 & 67,3 \\
\hline Total & 52 & 100,0 \\
\hline
\end{tabular}


Tabela 3 - Relação entre o diagnóstico Baixa autoestima situacional e suas características definidoras.

\begin{tabular}{|c|c|c|c|}
\hline \multirow{2}{*}{ Características definidoras } & \multicolumn{2}{|c|}{ Baixa autoestima situacional } & \multirow{2}{*}{ Estatística } \\
\hline & Presente & Ausente & \\
\hline \multicolumn{3}{|l|}{ 1. Comportamento indeciso } & \multirow{3}{*}{$\begin{array}{c}p=0,007^{*} \\
\operatorname{RP}=3,70 \\
\mathrm{IC} 95 \%(1,21-11,35)\end{array}$} \\
\hline Presente & 14 & 15 & \\
\hline Ausente & 3 & 20 & \\
\hline \multicolumn{3}{|l|}{ 2. Comportamento não assertivo } & \multirow{3}{*}{$\begin{array}{c}\mathrm{p}<0,001^{+} \\
\operatorname{RP}=3,75 \\
\mathrm{IC} 95 \%(1,86-7,56)\end{array}$} \\
\hline Presente & 9 & 3 & \\
\hline Ausente & 8 & 32 & \\
\hline \multicolumn{3}{|l|}{ 3. Expressões de desamparo } & \multirow{3}{*}{$\begin{array}{c}\mathrm{p}<0,001^{*} \\
\operatorname{RP}=6,89 \\
\mathrm{IC} 95 \%(2,25-21,05)\end{array}$} \\
\hline Presente & 14 & 7 & \\
\hline Ausente & 3 & 28 & \\
\hline \multicolumn{3}{|l|}{ 4. Expressões de sentimento de inutilidade } & \multirow{3}{*}{$\begin{array}{c}\mathrm{p}<0,001^{+} \\
\operatorname{RP}=8,02 \\
\mathrm{IC} 95 \%(3,11-20,66)\end{array}$} \\
\hline Presente & 13 & 2 & \\
\hline Ausente & 4 & 33 & \\
\hline \multicolumn{3}{|c|}{ 5. Relata verbalmente desafio situacional atual ao seu próprio valor } & \multirow{3}{*}{$\begin{array}{c}\mathrm{p}=0,980^{+} \\
\mathrm{RP}=1,02 \\
\mathrm{IC} 95 \%(0,2-5,32)\end{array}$} \\
\hline Presente & 1 & 2 & \\
\hline Ausente & 16 & 33 & \\
\hline \multicolumn{3}{|l|}{ 6. Verbalizações autonegativas } & \multirow{3}{*}{$\begin{array}{c}p<0,001^{*} \\
R P=4,94 \\
\text { IC95\% }(2,08-11,77)\end{array}$} \\
\hline Presente & 12 & 5 & \\
\hline Ausente & 5 & 30 & \\
\hline
\end{tabular}

*Teste de Qui-quadrado. ${ }^{+}$Teste exato de Fisher. $R P=$ Razão de prevalência

As características definidoras com frequência superior a 40\% foram: "Comportamento indeciso" $(55,8 \%)$ e "Expressões de desamparo" (40,4\%). A análise estatística evidenciou que gestantes que apresentavam a característica definidora "Comportamento não assertivo" também apresentavam idade gestacional média maior ( $p=0,009$ ), altura uterina também maior ( $p=0,023)$ e comparecimento a um maior número de consultas de pré-natal $(p=0,023)$. Estas duas últimas diferenças de médias estão diretamente relacionadas à idade gestacional. Com relação à característica "Relata verbalmente desafio situacional ao seu próprio valor" observou-se que sua presença estava relacionada a uma renda per capita média menor $(p=0,041)$. Gestantes que apresentavam a característica "Verbalizações autonegativas" apresentavam tempo médio de escolaridade $(p=0,034)$. As demais variáveis não apresentaram significância estatística. Para uma investigação mais aprofundada, procedeu-se a análise da associação entre o diagnóstico de interesse e cada característica definidora (Tabela 3).

Todas as características definidoras apresentaram associação estatisticamente significante com o diagnóstico de enfermagem ( $p<0,05)$, exceto "Relata verbalmente desafio situacional ao seu próprio valor" (Tabela 3). A razão de prevalência mostra que gestantes com "Comportamento indeciso" e "Comportamento não assertivo" apresentaram uma probabilidade três vezes maior de desenvolver Baixa autoestima situacional. Já a característica definidora "Verbalizações autonegativas" esteve associada a um aumento em quatro vezes da prevalência do diagnóstico. As características "Expressões de desamparo" e "Expressões de sentimento de inutilidade" se relacionaram a um aumento de seis e oito vezes, respectivamente, da probabilidade da presença do diagnóstico.

Com relação à análise da acurácia das características definidoras do diagnóstico de interesse, os melhores índices de sensibilidade foram obtidos pelas características "Comportamento indeciso" e "Expressões de desamparo", ambas com $82,35 \%$. Estas também apresentaram bom poder preditivo negativo. As características com maior especificidade foram "Expressões de sentimento de inutilidade" e "Relata verbalmente desafio situacional atual ao seu próprio valor", ambas com $94,29 \%$. O melhor poder de predição para a presença do diagnóstico (VPP) foi da característica "Expressões de sentimento de inutilidade" (86,67\%). Esta, também, foi a única que apresentou valores preditivos positivo e negativo elevados, área sob a curva ROC acima de $80 \%$ e odds ratio diagnóstica mais elevada $(44,39)$. Já as características "Comportamento indeciso", "Comportamento não assertivo", "Expressões de desamparo" e "Verbalizações autonegativas" se destacaram com valores negativos altos. 
Tabela 4 - Sensibilidade (S), Especificidade (E), Valor preditivo positivo (VVP), Valor preditivo negativo (VPN), Razão de verossimilhança positiva (RVP), Razão de verossimilhança negativa (RVN), Odds ratio diagnóstica (ORD), Acurácia (A) e Área sob a curva ROC (ROC) com os respectivos intervalos de confiança de $95 \%$ para as características definidoras do diagnóstico Baixa autoestima situacional.

\begin{tabular}{|c|c|c|c|c|c|c|}
\hline & \multicolumn{6}{|c|}{ Características definidoras } \\
\hline & 1 & 2 & 3 & 4 & 5 & 6 \\
\hline$S$ & $82,35[58,97-93,81]$ & $52,94[30,96-3,83]$ & $82,35[58,97-93,81]$ & $76,47[52,74-90,44]$ & $5,88[1,05-26,98]$ & $70,59[46,87-86,72]$ \\
\hline$E$ & $57,14[40,86-72,02]$ & $91,43[77,62-97,04]$ & $72,00[52,42-85,72]$ & $94,29[81,39-98,42]$ & $94,29[81,39-98,42]$ & $85,71[70,62-93,74]$ \\
\hline VPP & $48,28[31,39-65,57]$ & $75,00[46,77-91,11]$ & $66,67[45,37-82,81]$ & $86,67[62,12-96,26]$ & $33,33[6,15-79,23]$ & $70,59[46,87-86,72]$ \\
\hline VPN & $86,96[67,87-95,46]$ & $80,00[65,24-89,50]$ & $85,71[65,36-95,02]$ & $89,19[75,29-95,71]$ & $67,35[53,38-78,79]$ & $85,71[70,62-93,74]$ \\
\hline RVP & $1,92[1,21-3,06]$ & $6,18[1,99-19,21]$ & $2,94[1,50-5,75]$ & $13,38[3,41-52,48]$ & $1,03[0,25-4,29]$ & $4,94[2,10-11,65]$ \\
\hline RVN & $0,31[0,11-0,90]$ & $0,51[0,31-0,86]$ & $0,25[0,09-0,70]$ & $0,25[0,11-0,59]$ & $1,00[0,86-1,15]$ & $0,34[0,16-0,73]$ \\
\hline ORD & $5,82[1,54-30,27]$ & $10,96[2,57-62,25]$ & $10,85[2,58-61,99]$ & $44,39[8,62$ - 397,39] & $1,09[0,03-14,41]$ & $13,14[3,40-61,26]$ \\
\hline A & $65,38[51,80-76,85]$ & $78,85[65,97-87,76]$ & $76,19[61,47-86,52]$ & $88,46[77,03-94,60]$ & $65,38[51,80-76,85]$ & $80,77[68,10-89,20]$ \\
\hline ROC & 0,6975 & 0,7218 & 0,7718 & 0,8538 & 0,5008 & 0,7815 \\
\hline
\end{tabular}

1. Comportamento indeciso; 2. Comportamento não assertivo; 3. Expressões de desamparo; 4. Expressões de sentimento de inutilidade; 5 . Relata verbalmente desafio situacional atual ao seu próprio valor; 6 . Verbalizações autonegativas

\section{DISCUSSÃO}

O perfil sócio-demográfico da amostra estudada, composta por 52 gestantes, evidencia uma maior concentração de mulheres jovens, adolescentes, vivendo com companheiro, desempregadas, com escolaridade equivalente ao ensino médio incompleto e possuindo renda média de $R \$ 186,02$. Este perfil é típico das populações que habitam bairros da periferia das cidades brasileiras, onde se concentram famílias com baixo poder aquisitivo. Estima-se que, atualmente, 22,4\% das gestantes cadastradas no Sistema de Informação da Atenção Básica (SIAB) são adolescentes, em concordância com o presente estudo ${ }^{(14)}$.

Os achados referentes ao desemprego e à baixa renda podem ser correlacionados à idade das avaliadas, tendo em vista que prevaleceram adolescentes que eram estudantes. Estudos mostram que jovens, com menos de quatro anos de estudo, tendem a engravidar mais cedo e que o abandono dos estudos é frequente nestes $\operatorname{casos}^{(15)}$. Nestas situações, é comum que haja uma preocupação e uma mobilização dos familiares para resolver as adversidades, sobretudo quando o companheiro não tem disponibilidade para tal ${ }^{(16)}$.

Das gestantes avaliadas, $46,1 \%$ eram primigestas e $51,9 \%$ eram nulíparas. Estes resultados são representativos da redução da média do número de filhos por mulher na idade reprodutiva (taxa de fecundidade), fenômeno que vem ocorrendo no Brasil ${ }^{(14)}$.

O diagnóstico de enfermagem Baixa autoestima situacional esteve presente em $32,7 \%$ das gestantes. É notória a escassez de pesquisas na enfermagem relacionadas a este diagnóstico, de maneira que não foram encontrados estudos que identificassem a prevalência deste, nem de suas características definidoras.
O comportamento assertivo é uma característica relacionada à afirmação social e à capacidade de defender os próprios direitos e de expressar as ideias de maneira honesta ${ }^{(17)}$. Autores correlacionam o comportamento assertivo com uma alta autoestima ${ }^{(18)}$, o que vai ao encontro dos achados do presente estudo, visto que as gestantes que tinham a característica "Comportamento não assertivo" apresentaram uma probabilidade três vezes maior de desenvolver Baixa autoestima situacional.

A característica definidora citada anteriormente também mostrou relação com idade gestacional avançada, aumento da altura uterina e maior comparecimento às consultas de pré-natal. Este achado é explicável tendo em vista que são elementos que caracterizam um estágio mais avançado da gravidez, marcado por mudanças mais visíveis que podem repercutir negativamente na autoestima. Outros estudos apontam, de maneira similar, uma pior autoestima entre mulheres com 28 semanas de gestação ou mais ${ }^{(10,19)}$.

O valor próprio se baseia na percepção a respeito das próprias habilidades, realizações, posição social, recursos financeiros ou atributos físicos ${ }^{(17)}$. Esse conceito se relaciona com os resultados do presente estudo, visto que as mulheres que apresentaram a característica definidora "Relata verbalmente desafio situacional atual ao seu próprio valor" demonstraram menor renda per capita. Ademais, essa característica apresentou uma elevada especificidade.

Conforme apresentado nos resultados, a característica "Expressões de sentimento de inutilidade" apresentou os melhores valores entre os vários índices estudados. Esta característica deve ser considerada como uma das que melhor podem auxiliar o enfermeiro na detecção de baixa autoestima em gestantes.

As "Verbalizações autonegativas" se destacaram entre as gestantes com menor escolaridade e representaram um aumento em quatro vezes da prevalência de Baixa autoestima 
situacional. A baixa escolaridade é considerada um dos principais fatores de risco para a depressão gestacional, por estar relacionada, na maioria das vezes, a um cenário de pobreza, falta de apoio, despreparo e abandono por parte do parceiro, acarretando problemas de ordem psicológica, afetiva e social $^{(20)}$. Neste ponto, a característica definidora "Expressões de desamparo" apresentou elevada sensibilidade e valor preditivo negativo alto, além de aumentar em seis vezes a probabilidade do desenvolvimento do diagnóstico estudado.

A característica definidora "Comportamento indeciso" foi a mais prevalente, fato que chamou atenção, tendo em vista que $88,5 \%$ das gestantes apresentavam um relacionamento estável, contrariando o que a literatura aponta, isto é, que relacionamentos instáveis aumentam a indecisão das gestantes ${ }^{(21)}$. É importante salientar que esta característica demonstrou alta sensibilidade e representou um aumento em três vezes da probabilidade do desenvolvimento de Baixa autoestima situacional.

Por fim, a literatura aponta que mulheres com baixa autoestima têm 39 vezes mais chances de apresentar sintomas depressivos quando comparadas a mulheres com alta autoestima $^{(22)}$. Portanto, pesquisas que avaliem a autoestima em gestantes são de suma importância, como medidas de prevenção da depressão pós-parto.

Neste ponto, vale ressaltar a importância do enfermeiro, enquanto membro da equipe de saúde da família, na identificação das situações de baixa autoestima e na oferta de suporte às gestantes com esta problemática. É inerente à consulta de enfermagem a investigação das respostas do organismo materno à gestação e dos problemas reais ou potenciais, ações que são potencializadas quando há boa interação, confiança e credibilidade durante a assistência ${ }^{(23)}$.

\section{CONCLUSÃO}

O estudo possibilitou identificar a prevalência do diagnóstico de enfermagem Baixa autoestima situacional em gestantes e a acurácia das características definidoras deste (mediante a avaliação da sensibilidade e da especificidade); e subsidiou a avaliação do uso da escala de Rosenberg como ferramenta para a identificação do referido diagnóstico.

Os resultados podem contribuir com a identificação de problemas psicossociais durante o pré-natal. Considerando-se que o estudo foi realizado apenas com gestantes, as informações encontradas devem ser vistas com parcimônia. Além disso, ainda é pouco conhecida a real influência de muitas características definidoras como preditores deste diagnóstico.

As gestantes adolescentes e com menor nível econômico são, notadamente, susceptíveis a complicações psicológicas como a Baixa autoestima situacional. Este fato mostra que pesquisas sobre intervenções de enfermagem direcionadas a este diagnóstico são particularmente importantes. A identificação de características definidoras acuradas é fundamental para uma sistematização eficaz da assistência, visto que contribui para tornar os diagnósticos da Taxonomia NANDA-I mais claros, facilitando o uso destes pelos enfermeiros.

\section{REFERÊNCIAS}

1. Piccinini CA, Lopes RS, Gomes AG, Nardi T. Gestação e a constituição da maternidade. Psicol Estud 2008;13(1):63-72

2. Lowdermilk DL, Perry SE, Bobsk IM. O Cuidado em Enfermagem Materna. 5.ed. Porto Alegre: Artmed; 2002. p. 928.

3. Wadhwa PD, Dunkel-Schetter C, Chicz-Demet A, Porto $M$, Sandman CA. Prenatal psychosocial factors and the neuroendocrine axis in human pregnancy. Psychosom Med 1996;58(5):432-46.

4. North American Nursing Diagnosis Association. Diagnósticos de enfermagem da NANDA: definições e classificação 2009-2011. Porto Alegre: Artmed; 2010. p. 456.

5. Moreira AV, Arcanjo MZ, Tavares PIL, Lopes MVO. Estudo do diagnóstico de enfermagem fadiga em gestantes atendidas numa unidade básica de atenção à saúde. Acta Paul Enferm 2006;19(1):70-5.

6. Gouveia HG, Lopes MHBM. Diagnósticos de enfermagem e problemas colaborativos mais comuns na gestação de risco. Rev Latino-Am Enferm 2004;12(2):175-82.

7. Romano A, Negreiros J, Martins T. Contributos para a validação da escala de auto-estima de Rosenberg numa amostra de adolescentes da região interior do Norte do país. Psicol Saúde Doenças 2007;8(1):109-16.
8. Avanci JQ, Assis SG, Santos NC, Oliveira RVC. Adaptação transcultural da escala de auto-estima para adolescentes. Psicol Reflex Crít 2007;20(3):397-405.

9. Rosenberg M. Society and the adolescent self-image. Princeton: Princeton University Press; 1965. p. 326.

10. Dias MS, Silva RA, Souza LDM, Lima RC, Pinheiro RT, Moraes IGS. Auto-estima e fatores associados em gestantes da cidade de Pelotas, Rio Grande do Sul, Brasil. Cad Saúde Pública 2008;24(12):2787-97.

11. Souza DBL, Ferreira MC. Autoestima pessoal e coletiva em mães e não mães. Psicol Estud 2005;10(1):19-25.

12. Lunney M. Critical thinking and accuracy of nurses' diagnoses - Part I: risk of low accuracy diagnoses and new views of critical thinking. Rev Esc Enferm USP 2003;37(2):17-24.

13. Rosen RC, Brown C, Heiman J, Leiblum S, Meston CM, Shabsigh $R$, et al. The female sexual function index (FSFI): a multidimensional self-report instrument for the assessment of female sexual function. J Sex Marital Ther 2000;2:191-208.

14. Ministério da Saúde [homepage na internet] Sistema de Informação da Atenção Básica - SIAB. [acesso em 5 out 2009]. Disponível em: < http://siab.datasus.gov.br/SIAB/ index.php?area $=04 \mathrm{~A} 01$ \&item $=1>$ 
15. Souza VLC, Corrêa MSM, Souza SL, Beserra MA. O aborto entre adolescentes. Rev Latino-Am Enferm 2001;9(2):42-7.

16. Silva L, Tonete VLP. A gravidez na adolescência sob a perspectiva dos familiares: compartilhando projetos de vida e cuidado. Rev Latino-Am Enferm 2006;14(2):199-206.

17. DelPrette ZAP, DelPrette A. Psicologia das habilidades sociais: terapia e educação. Petrópolis: Vozes; 1999. p. 208.

18. Bandeira DR, Arteche AX, Reppold CT. Escala de autopercepção de harter para adolescentes: um estudo de validação. Psicol Teor Pesqui 2008;24(3):341-5.

19. Marcon SS. Vivenciando a gravidez: processos e sub-processos de uma teoria fundamentada nos dados. Rev Latino-Am Enferm 1995;3(2):165-79.
20. Pereira PK, Lovisi GM. Prevalência da depressão gestacional e fatores associados. Revista Psiquiatr Clín 2008;35(4):144-53.

21. Carvalho EC, Hartmann JB. Feridas abertas: identificando sentimentos maternos em grávidas desamparadas. Rev Saúde Pesqui 2009;2(1):69-80.

22. Hall LA, Kotch JB, Browne D, Rayens MK. Self-esteem as a mediator of the effects of stressors and social resources on depressive symptoms in postpartum mothers. Nurs Res 1996;45(4):231-8.

23. Pereira SVM, Bachion MM. Diagnósticos de Enfermagem identificados em gestantes durante o pré-natal. Rev Bras Enferm 2005;58(6):659-64. 